\title{
Nasopharyngeal carcinoma incidence and mortality in China, 2013
}

\author{
Kuang-Rong Wei ${ }^{1}$, Rong-Shou Zheng ${ }^{2}$, Si-Wei Zhang ${ }^{2}$, Zhi-Heng Liang ${ }^{1}$, Zhu-Ming Li ${ }^{1}$ and Wan-Qing Chen ${ }^{2 *}$
}

\begin{abstract}
Background: We estimated the incidence and mortality of nasopharyngeal carcinoma (NPC) in China in 2010 according to the data of 145 domestic population-based cancer registries in 2014, and no such reports since then. Hence, to further and better understand its epidemiology in China and to provide more precise scientific information for its control and prevention in China, we analyzed the NPC incidence and mortality of 255 domestic populationbased cancer registries, and estimated the national rates in 2013 again.

Methods: NPC incidence and mortality data of 255 domestic cancer registries in 2013, accepted by the 2016 National Cancer Registry Annual Report, were collected and collated, and the indices of NPC such as the numbers of new cases and deaths, crude rates, age-standardized rates, and truncated rates of incidence and mortality were calculated and analyzed. The incidence and mortality in China and its constituent areas were estimated according to the national population in 2013.
\end{abstract}

Results: An estimated 42,100 new cases and 21,320 deaths were attributed to NPC in China in 2013, accounting for $1.14 \%$ of all new cancer cases and $0.96 \%$ of all cancer-related deaths that year in China. Crude incidence and mortality of NPC were $3.09 / 100,000$ and 1.57/100,000, respectively. World age-standardized incidence and mortality were $2.17 / 100,000$ and $1.08 / 100,000$, respectively. The incidence and mortality of males were obviously higher than those of females and slightly higher in urban areas than in rural areas. Among seven Chinese administrative regions, NPC incidence and mortality were obviously higher in South China than in other regions and lowest in North China. Top 3 incidence and mortality provinces and registering areas all located in South China. The age-specific incidence and mortality rose quickly from age 25-29 and 35 to 39 years, respectively, peaked at different ages and varied by location.

Conclusions: These results demonstrated that NPC incidence and mortality in China in 2013 were also at high levels worldwide, which suggested that its control and prevention should be enhanced.

Keywords: Nasopharyngeal carcinoma, Incidence, Mortality, China

In 2014, for the first time in China, we estimated NPC incidence and mortality in whole China according to the data of 145 domestic population-based cancer registries with high data quality and the national population in 2010 [1], and we could know more about the epidemiology of NPC in China, except the data of GLOBOCAN [2], Cancer Incidence in Five Continents (CIF) [3], World Health Organization (WHO) [4], the national alldeath-causes retrospective sampling survey [5], and the

\footnotetext{
*Correspondence: chenwq@cicams.ac.cn

${ }^{2}$ National Central Cancer Registry, National Cancer Center, Beijing 100021,

P. R. China

Full list of author information is available at the end of the article
}

domestic cancer registries $[1,6]$. At the same time, some researches revealed that, during the past decade, incidence of NPC is gradually declining worldwide, even in endemic regions, and mortality has fallen substantially [7-10], although its incidence increased in some countries [11] and tumor histology such as non-keratinizing tumors [12]. The mortality in China had been steadily declining and would continue to drop in the next few years [13], but some high-risk areas in China witnessed different trends, such as an increasing death trend in Guangxi autonomous region [14], declined incidence and mortality in urban Guangzhou [15], a stable incidence in Sihui [16] and Zhongshan [17], and a declining death 
trend in Zhongshan [18]. As of now, the incidence and mortality data of NPC in whole China were few. Hence, to further and better understand its epidemiological situation in China and to provide more precise scientific information for its control and prevention, we estimated the incidence and mortality of NPC in China in 2013 again according to the data of 255 domestic populationbased cancer registries.

\section{Data and methods}

\section{Data source}

The NPC incidence and mortality data in this study came from 255 domestic population-based cancer registries, whose data quality met the criteria of National Central Cancer Registry (NCCR) [the percentage of cases with morphological verification (MV\%) $>66 \%$, the percentage of cases with death certification only $(\mathrm{DCO} \%)<15 \%$, mortality to incidence $[\mathrm{M} / \mathrm{I}]$ ratio between 0.6 and 0.8 , and the percentage of the diagnosis of unknown basis $(\mathrm{UB} \%)<5 \%$ ] and accepted by the 2016 National Cancer Registry Annual Report. The 255 registries, including 88 in urban areas and 167 in rural areas, were distributed in 31 provinces, autonomous regions, and municipalities directly under central government of China [19, 20]. NPC is coded as C11 in the International Statistical Classification of Diseases and Related Health Problems 10th Revision (ICD-10) [21].

Population data came from the Statistical and/or Public Security Bureaus of the above mentioned cancer registering areas, which covered a population of $226,494,490$ persons, including 114,860,339 (50.71\%) males and $111,634,151$ (49.29\%) females, or $111,595,772$ (49.27\%) persons in urban and $114,898,718$ (50.73\%) in rural areas. The covered population accounted for $16.65 \%$ of total Chinese population by the end of 2013 .

\section{Statistical indices and methods}

Statistical indices included the numbers of NPC incident cases and deaths, proportions, crude rates, age-standardized rates (ASRs) by Chinese (ASRC) and world (ASRW) standard population (ASIRC and ASMRC represents the ASRC of incidence and mortality, respectively, and ASIRW and ASMRW represents the ASRW of incidence and mortality, respectively), cumulative rates (aged 0-74 years), truncated age-standardized rates (TASR) (aged 35-64 years), and age-specific rates, stratified by areas, regions, and genders. The statistical methods recommended by the Guideline for Chinese Cancer Registration [22] were adopted. The standard population of China in 2000 and that of the World in 1985 (Segi) were used to calculate ASRs $[19,20]$.

The cancer registering areas included in the present study were classified as urban and rural areas or Eastern, Middle, and Western areas and were divided into seven administrative regions: South China, North China, Central China, East China, Southwest China, Northwest China, and Northeast China according to the criteria of the National Bureau of Statistics [19]. The statistical indices were calculated for the above areas and regions. By multiplying the age-specific incidence and mortality with the population numbers of corresponding age groups in 2013 in above each stratification, NPC incident case and death numbers in each stratification were obtained, and the estimated incident cases and deaths counts in whole China were obtained by pooling the data $[19,20]$.

All statistical analysis was conducted by using SAS software (SAS Institute Inc., Cary, USA).

\section{Results}

Data quality

Data quality varied by cancer registering area in this study. Data quality in urban areas was higher than that in rural areas, with the data quality in males close to that in females. The data quality was the lowest for the registries in the Western area, and similar for the registries in the Middle and Eastern areas. The MV\%, DCO\%, M/I ratio, and UB\% of NPC data from the above mentioned 255 registries were $75.12,1.18,0.52$, and $0.65 \%$, respectively; they were $77.20,1.09,0.51$, and $0.35 \%$, respectively, for urban registries, and $72.78 \%, 1.29 \%, 0.54 \%$, and $0.99 \%$, respectively, for rural registries (Table 1).

\section{Incidence estimation}

About 42,100 new NPC cases, accounting for 1.14\% of all new cancer cases in China in 2013, were estimated to occur in China that year; its crude incidence, ASIRC, and ASIRW were 3.09/100,000, 2.31/100,000, and 2.17/100,000, respectively. About 30,000 new male NPC cases, accounting for $1.47 \%$ of all new male cancer cases in China in 2013, were estimated to occur in China that year; its crude incidence, ASIRC, and ASIRW were $4.31 / 100,000,3.26 / 100,000$, and $3.07 / 100,000$, respectively. About 12,000 new female NPC cases, accounting for $0.74 \%$ of all new female cancer cases in China in 2013, were estimated to occur in China that year; its crude incidence, ASIRC, and ASIRW were 1.81/100,000, $1.35 / 100,000$, and $1.25 / 100,000$, respectively. The male incidence was higher than the female incidence. The ASIRW of males in urban, rural, and all areas were 2.59, 2.29 , and 2.46 times higher than those of females, respectively. The incidence in the Western area was the highest, followed by those in the Middle and Eastern areas; the incidence in the Middle area was slightly higher than that in the Eastern area (Table 2). 
Table 1 Quality of nasopharyngeal carcinoma (NPC) data in 2013 from 255 Chinese cancer registries

\begin{tabular}{llllll}
\hline Areas & Sex & M/I ratio & MV\% & DCO\% & UB\% \\
\hline All & Both & 0.52 & 75.12 & 1.18 & 0.65 \\
& Male & 0.54 & 75.02 & 1.25 & 0.69 \\
Urban areas & Female & 0.48 & 75.36 & 1.02 & 0.53 \\
& Both & 0.51 & 77.20 & 1.09 & 0.35 \\
& Male & 0.53 & 77.36 & 1.26 & 0.37 \\
Rural areas & Female & 0.45 & 76.78 & 0.66 & 0.28 \\
& Both & 0.54 & 72.78 & 1.29 & 0.99 \\
& Male & 0.55 & 72.31 & 1.24 & 1.07 \\
Eastern area & Female & 0.50 & 73.88 & 1.40 & 0.80 \\
& Both & 0.46 & 75.62 & 1.31 & 1.76 \\
& Male & 0.47 & 75.98 & 1.39 & 1.83 \\
Middle area & Female & 0.45 & 74.82 & 1.12 & 1.57 \\
& Both & 0.46 & 75.74 & 2.15 & 0.22 \\
& Male & 0.47 & 75.73 & 2.01 & 0.16 \\
& Female & 0.42 & 75.85 & 2.20 & 0.34 \\
Western area & Both & 0.52 & 63.62 & 3.33 & 1.02 \\
& Male & 0.56 & 63.56 & 3.61 & 0.70 \\
& Female & 0.45 & 63.74 & 2.72 & 1.59 \\
\hline
\end{tabular}

M/I mortality to incidence, $M V \%$ the percentage of cases with morphologically verification, $D C O \%$ the percentage of cases with death certification only, UB\% the percentage of diagnosis of unknown basis

\section{Age-specific incidence}

NPC age-specific incidences in China in 2013 began to increase quickly from age 25-29 years, peaked at age 60-64 for males and 75-79 years for females, and decreased obviously thereafter. The age-specific incidence in males was much higher than that in females. There was no obvious difference in the trends of male and female age-specific incidences between urban and rural areas (Fig. 1). Age-specific incidence varied by area at some degree, e.g., it peaked at age 80-84 years for males in the Western area and at age $85+$ years for females in the Eastern area.

\section{Incidence differences between regions}

An estimated 24,300 new NPC cases, accounting for $1.17 \%$ of all new cancer cases in Chinese urban areas in 2013, occurred in Chinese urban areas that year; the crude incidence, ASIRC, and ASIRW were 3.33/100,000, $2.40 / 100,000$, and $2.23 / 100,000$, respectively. An estimated 17,700 new NPC cases, accounting for $1.10 \%$ of all new cancer cases in Chinese rural areas in 2013, occurred in Chinese rural areas that year; the crude incidence, ASIRC, and ASIRW were 2.82/100,000, 2.19/100,000, and $2.07 / 100,000$, respectively (Table 2 ). The incidences in urban areas were slightly higher than those in rural areas. The crude incidence, ASIRC, and ASIRW in urban areas were only $1.18,1.10$, and 1.03 times higher than those in rural areas, respectively.

Generally, the highest incidence was seen in the Western area, followed by the Middle and Eastern areas in China in 2013; the same trend was found in rural areas. In urban areas, the highest rate was seen in Western

Table 2 NPC incidence in China in 2013

\begin{tabular}{|c|c|c|c|c|c|c|c|c|}
\hline Areas & Sex & $\mathrm{CR}\left(1 / 10^{5}\right)$ & Prop (\%) & $\operatorname{ASIRC}\left(1 / 10^{5}\right)$ & ASIRW $\left(1 / 10^{5}\right)$ & Cum rate (\%) & $\operatorname{TASR}\left(1 / 10^{5}\right)$ & Rank \\
\hline \multirow[t]{3}{*}{ All } & Both & 3.09 & 1.14 & 2.31 & 2.17 & 0.24 & 4.88 & 19 \\
\hline & Male & 4.31 & 1.47 & 3.26 & 3.07 & 0.34 & 7.01 & 14 \\
\hline & Female & 1.81 & 0.74 & 1.35 & 1.25 & 0.13 & 2.68 & 19 \\
\hline \multirow[t]{3}{*}{ Urban areas } & Both & 3.33 & 1.17 & 2.40 & 2.23 & 0.24 & 5.09 & 20 \\
\hline & Male & 4.73 & 1.58 & 3.43 & 3.21 & 0.35 & 7.37 & 14 \\
\hline & Female & 1.87 & 0.70 & 1.35 & 1.24 & 0.13 & 2.71 & 19 \\
\hline \multirow[t]{3}{*}{ Rural areas } & Both & 2.82 & 1.11 & 2.19 & 2.07 & 0.23 & 4.59 & 19 \\
\hline & Male & 3.82 & 1.33 & 3.04 & 2.89 & 0.32 & 6.50 & 12 \\
\hline & Female & 1.75 & 0.79 & 1.34 & 1.26 & 0.14 & 2.65 & 20 \\
\hline \multirow[t]{3}{*}{ Eastern area } & Both & 2.93 & 0.96 & 2.07 & 1.93 & 0.21 & 5.52 & 19 \\
\hline & Male & 4.14 & 1.23 & 2.94 & 2.76 & 0.31 & 8.17 & 14 \\
\hline & Female & 1.71 & 0.61 & 1.21 & 1.11 & 0.12 & 2.67 & 20 \\
\hline \multirow[t]{3}{*}{ Middle area } & Both & 2.89 & 1.15 & 2.25 & 2.15 & 0.24 & 4.77 & 21 \\
\hline & Male & 3.98 & 1.42 & 3.16 & 3.02 & 0.34 & 6.47 & 15 \\
\hline & Female & 1.74 & 0.78 & 1.33 & 1.26 & 0.14 & 2.95 & 20 \\
\hline \multirow[t]{3}{*}{ Western area } & Both & 4.57 & 1.86 & 3.58 & 3.37 & 0.37 & 6.58 & 18 \\
\hline & Male & 6.35 & 2.25 & 5.03 & 4.73 & 0.52 & 8.78 & 14 \\
\hline & Female & 2.72 & 1.32 & 2.12 & 1.99 & 0.21 & 4.31 & 19 \\
\hline
\end{tabular}

CR crude rate, Prop proportion, ASIRC age-standardized incidence rate by 2000 Chinese standard population, $A S I R W$ age-standardized incidence rate by 1985 Segi's world standard population, Cum rate cumulative rate for patients aged 0-74 years, TASR truncated age-standardized rate for patients aged 35-64 years, Rank rank of NPC incidence in all cancers' incidences 


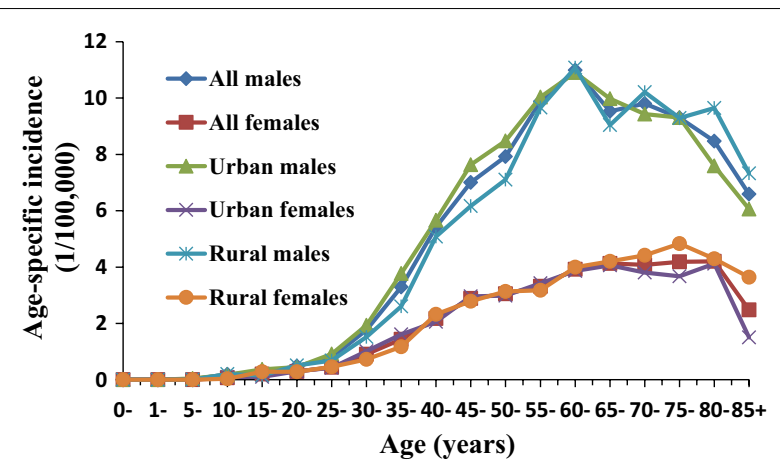

Fig. 1 Nasopharyngeal carcinoma (NPC) age-specific incidence in China in 2013. The age-specific incidence increases remarkably from age 25-29 years, peaks at age 60-64 for males and age 75-79 for females, and decreases obviously thereafter. The incidences in males are much higher than those in females, but no obvious difference exists in age-specific incidence in either males or females between urban and rural areas

areas, followed by Eastern and Middle areas. However, no significant difference existed for the incidence among the above three areas.

Among the Chinese seven administrative regions, the incidence in South China was the highest, followed by those in Southwest, Central, East, Northwest, Northeast, and North China. Notably, the ASIRW in South China $(9.69 / 100,000)$ was 3.4 times higher than that in Southwest China $(2.85 / 100,000)$, in which the incidence was the second highest.

In terms of ASIRW, top 3 incidence provinces among 31 Chinese provinces, autonomous regions, and municipalities directly under the central government in 2013 were Guangxi, Guangdong, and Hunan, whose ASIRWs were $11.16 / 100,000,10.38 / 100,000$, and 5.38/100,000, respectively, all located in South China. Tibet, Sichuan, Chongqing, and Hubei also ranked top 10 incidence provinces, which do not locate in South China (Table 3). Top 3 incidence areas in the 255 domestic cancer registries in 2013 were Cangwu county of Guangxi autonomous region, Sihui city of Guangdong province, and Longnan county of Jiangxi province, whose ASIRWs were $25.39 / 100,000,18.74 / 100,000$, and 15.52/100,000, respectively. Top 10 incidence areas all located in South China (Table 4).

\section{Mortality estimation}

About 21,320 NPC death cases, accounting for $0.96 \%$ of all cancer death cases in 2013 in China, were estimated to occur in China that year; its crude mortality, ASMRC, and ASMRW were $1.57 / 100,000,1.10 / 100,000$, and $1.08 / 100,000$, respectively. About 15,730 male NPC death cases, accounting for $1.12 \%$ of all male cancer death cases in 2013 in China, were estimated to occur in China that year. The crude mortality, ASMRC, and ASMRW were $2.26 / 100,000,1.63 / 100,000$, and $1.60 / 100,000$, respectively. About 5580 female NPC death cases, accounting for $0.68 \%$ of all female cancer death cases in 2013 in China, were estimated to occur in China that year. The crude mortality, ASMRC, and ASMRW were $0.84 / 100,000,0.58 / 100,000$, and $0.56 / 100,000$, respectively. The male mortality was much higher than the female mortality (Table 5). The ASMRW in urban, rural, and both areas in males were 3.12, 2.59, and 2.86 times of those in females, respectively. The mortality in the Western area was the highest, followed by those in the Middle and Eastern areas, with the mortality in the Middle area slightly higher than that in the Eastern area.

Table 3 Top 10 NPC incidence and mortality provinces, autonomous regions, and municipalities in China, 2013

\begin{tabular}{|c|c|c|c|c|c|c|c|c|}
\hline \multirow[t]{2}{*}{ Rank } & \multicolumn{4}{|c|}{ Incidence $\left(1 / 10^{5}\right)$} & \multicolumn{4}{|c|}{ Mortality $\left(1 / 10^{5}\right)$} \\
\hline & Province $^{a}$ & CR & ASIRC & ASIRW & Province $^{a}$ & CR & ASMRC & ASMRW \\
\hline 1 & Guangxi & 12.37 & 11.16 & 10.49 & Guangxi & 5.83 & 5.16 & 5.01 \\
\hline 2 & Guangdong & 12.30 & 10.38 & 9.62 & Guangdong & 6.26 & 5.06 & 4.99 \\
\hline 3 & Hunan & 6.97 & 5.38 & 5.08 & Hainan & 4.44 & 3.27 & 3.27 \\
\hline 4 & Jiangxi & 5.62 & 5.08 & 4.88 & Hunan & 3.76 & 2.88 & 2.71 \\
\hline 5 & Hainan & 5.45 & 3.90 & 3.83 & Jiangxi & 2.89 & 2.53 & 2.44 \\
\hline 6 & Fujian & 4.69 & 3.87 & 3.65 & Guizhou & 3.04 & 2.40 & 2.38 \\
\hline 7 & Tibet & 2.66 & 3.15 & 3.06 & Fujian & 2.58 & 2.08 & 2.05 \\
\hline 8 & Sichuan & 4.35 & 3.21 & 3.05 & Sichuan & 2.15 & 1.52 & 1.49 \\
\hline 9 & Chongqing & 4.41 & 3.12 & 2.92 & Shanghai & 3.13 & 1.38 & 1.37 \\
\hline 10 & Hubei & 4.46 & 3.04 & 2.88 & Hubei & 1.97 & 1.25 & 1.25 \\
\hline
\end{tabular}

\footnotetext{
a Including provinces, autonomous regions, and municipalities directly under central government of China
} 
Table 4 Top 10 NPC incidence and mortality areas in China, $2013\left(1 / 10^{5}\right)$

\begin{tabular}{|c|c|c|c|c|c|c|c|c|}
\hline \multirow[t]{2}{*}{ Rank } & \multicolumn{4}{|l|}{ Incidence } & \multicolumn{4}{|l|}{ Mortality } \\
\hline & Area & CR & ASIRC & ASIRW & Area & CR & ASMRC & ASMRW \\
\hline 1 & Cangwu county of Guangxi & 27.13 & 26.95 & 25.39 & Sihui city of Guangdong & 20.19 & 14.28 & 14.24 \\
\hline 2 & Sihui city of Guangdong & 25.26 & 20.91 & 18.74 & Mayang county of Hunan & 14.09 & 13.06 & 12.54 \\
\hline 3 & Longnan county of Jiangxi & 14.42 & 16.04 & 15.52 & Cangwu county of Guangxi & 12.2 & 11.22 & 11.16 \\
\hline 4 & Zhongshan city of Guangdong & 17.54 & 14.31 & 13.06 & Zhongshan city of Guangdong & 9.82 & 8.48 & 8.54 \\
\hline 5 & Nanxiong city of Guangdong & 13.81 & 11.79 & 11.22 & Longnan county & 9.34 & 7.79 & 7.56 \\
\hline 6 & Jiangmen urban area of Guangdong & 14.99 & 11.54 & 10.64 & Fushui county of Guangxi & 2.44 & 6.98 & 6.42 \\
\hline 7 & Mayang county of Hunan & 14.77 & 10.94 & 10.53 & Jiangmen urban area of Guangdong & 9.31 & 6.34 & 5.96 \\
\hline 8 & Beiliu city of Guangxi & 11.04 & 11.24 & 10.27 & Hepu county of Guangxi & 5.7 & 5.81 & 5.55 \\
\hline 9 & Fushui county of Guangxi & 11.58 & 10.92 & 10.23 & Beiliu city of Guangxi & 3.87 & 4.50 & 4.50 \\
\hline 10 & Hepu county of Guangxi & 11.74 & 10.34 & 9.96 & Nanxiong city of Guangdong & 9.57 & 4.32 & 4.31 \\
\hline
\end{tabular}

$C R$ crude rate, $A S I R C$ age-standardized incidence rate by 2000 Chinese standard population, $A S I R W$ age-standardized incidence rate by 1985 Segi's world standard population, ASMRC age-standardized mortality rate by 2000 Chinese standard population, ASMRW age-standardized mortality rate by 1985 Segi's world standard population

Table 5 NPC mortality in China in 2013

\begin{tabular}{|c|c|c|c|c|c|c|c|c|}
\hline Areas & Sex & $C R\left(1 / 10^{5}\right)$ & Prop (\%) & ASMRC $\left(1 / 10^{5}\right)$ & ASMRW $\left(1 / 10^{5}\right)$ & Cum rate (\%) & $\operatorname{TASR}\left(1 / 10^{5}\right)$ & Rank \\
\hline \multirow[t]{3}{*}{ All } & Both & 1.57 & 0.96 & 1.10 & 1.08 & 0.12 & 2.09 & 18 \\
\hline & Male & 2.26 & 1.12 & 1.63 & 1.60 & 0.19 & 3.10 & 13 \\
\hline & Female & 0.84 & 0.68 & 0.58 & 0.56 & 0.06 & 1.06 & 18 \\
\hline \multirow[t]{3}{*}{ Urban areas } & Both & 1.61 & 1.00 & 1.09 & 1.07 & 0.12 & 2.10 & 19 \\
\hline & Male & 2.38 & 1.21 & 1.65 & 1.62 & 0.19 & 3.16 & 14 \\
\hline & Female & 0.81 & 0.65 & 0.54 & 0.52 & 0.06 & 1.01 & 19 \\
\hline \multirow[t]{3}{*}{ Rural areas } & Both & 1.51 & 0.91 & 1.12 & 1.09 & 0.13 & 2.08 & 18 \\
\hline & Male & 2.12 & 1.02 & 1.62 & 1.58 & 0.18 & 3.02 & 12 \\
\hline & Female & 0.87 & 0.71 & 0.63 & 0.61 & 0.07 & 2.13 & 16 \\
\hline \multirow[t]{3}{*}{ Eastern areas } & Both & 1.62 & 0.86 & 1.02 & 1.00 & 0.12 & 3.27 & 17 \\
\hline & Male & 2.35 & 1.01 & 1.51 & 1.49 & 0.18 & 0.95 & 13 \\
\hline & Female & 0.87 & 0.61 & 0.53 & 0.52 & 0.06 & 2.02 & 18 \\
\hline \multirow[t]{3}{*}{ Middle areas } & Both & 1.41 & 0.91 & 1.06 & 1.03 & 0.11 & 2.98 & 19 \\
\hline & Male & 2.05 & 1.06 & 1.59 & 1.55 & 0.17 & 1.03 & 13 \\
\hline & Female & 0.75 & 0.64 & 0.54 & 0.53 & 0.06 & 3.21 & 18 \\
\hline \multirow[t]{3}{*}{ Western areas } & Both & 2.15 & 1.38 & 1.63 & 1.59 & 0.18 & 4.82 & 15 \\
\hline & Male & 3.07 & 1.54 & 2.36 & 2.29 & 0.27 & 1.55 & 10 \\
\hline & Female & 1.20 & 1.07 & 0.91 & 0.89 & 0.10 & 2.13 & 15 \\
\hline
\end{tabular}

CR crude rate, Prop proportion, ASMRC age-standardized mortality rate by Chinese standard population, ASMRW, age-standardized mortality rate by 1985 Segi's world standard population, Cum rate cumulative rate for patients aged 0-74 years, TASR truncated age-standardized rate for patients aged 35-64 years, Rank rank of NPC mortality in all cancers' mortalities

\section{Age-specific mortality}

NPC age-specific mortalities in China in 2013 began to rise quickly from age 35-39 years, peaked at age 80-84 years for males and at age $85+$ years for females, and down thereafter. The male mortality was obviously higher than the female mortality. The trends of male and female age-specific mortalities in urban and rural areas were basically the same, with only slight difference, such as the urban male and rural female mortalities peaked at age 75-79 years (Fig. 2). The age-specific mortalities of $\mathrm{NPC}$ varied at some degree in different Chinese areas, e.g., it peaked at age 65-69 years for males in the Western area and at 75-79 years for females in the Middle area.

\section{Mortality differences between regions}

About 11,780 NPC death cases, accounting for $1.00 \%$ of all cancer death cases in Chinese urban areas in 2013, were estimated to occur in Chinese urban areas that 


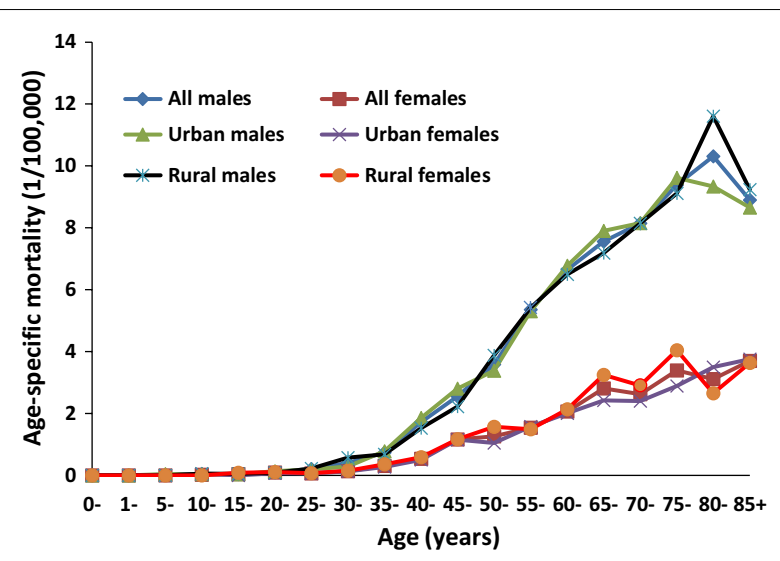

Fig. 2 NPC age-specific mortality in China in 2013. The age-specific mortality rises quickly from age 35-39 years, peaks at age 80-84 for males and $85+$ years for females. The mortalities in males are much higher than those in females. The patterns of male and female agespecific mortalities in urban and rural areas are basically the same

year. The crude mortality, ASMRC, and ASMRW were $1.61 / 100,000,1.09 / 100,000$, and $1.07 / 100,000$, respectively. About 9540 NPC death cases, accounting for $0.91 \%$ of all cancer-related death cases in Chinese rural areas in 2013, were estimated to occur in Chinese rural areas that year. The crude mortality, ASMRC, and ASMRW were $1.51 / 100,000,1.12 / 100,000$, and $1.09 / 100,000$, respectively (Table 3 ). The mortalities in urban areas were close to those in rural areas, with the crude mortality slightly higher but the ASMRC and ASMRW slightly lower in urban areas (Table 5).

Generally, the highest mortality was seen in the Western area, followed by those in the Middle and Eastern areas in China in 2013, but with no significant difference; the same trend was found in rural areas. However, in urban areas, the highest rates were seen in the Western area, followed by the Eastern and Middle areas, although without significant difference too.

Among the Chinese seven administrative regions, the mortality in South China was the highest, followed by those in Southwest, Central, East, Northwest, Northeast, and North China. Notably, the ASMRW $(4.92 / 100,000)$ in South China was 3.8 times higher than that in Southwest China $(1.31 / 100,000)$, in which the mortality was the second highest.

In terms of ASMRW, top 3 death provinces in the 31 provinces, autonomous regions, and municipalities directly under the central government in China in 2013 were Guangxi, Guangdong, and Hainan, in which the ASMRWs were $5.01 / 100,000,4.99 / 100,000$, and $3.27 / 100,000$, respectively, all located in South China. Guizhou, Sichuan, Chongqing, Shanghai, and Hubei also ranked the top 10 mortality provinces, which do not locate in South China (Table 3). Top 3 mortality areas in the 255 domestic cancer registries in 2013 were Cangwu county of Guangxi autonomous region, Sihui city of Guangdong province, and Longnan county of Jiangxi province, in which the ASMRWs were 14.24/100,000, $12.54 / 100,000$, and $11.16 / 100,000$, respectively. Top 10 mortality areas all located in South China.

\section{Discussions}

Compared with the data of GLOBOCAN 2012 [2], NPC incidence and mortality in China in 2013 (ASIRW and ASMRW: $2.31 / 100,000$ and $1.10 / 100,000$, respectively) were lower than those in Southeast Asia $(4.3 / 100,000$ and $2.5 / 100,000$, respectively), significantly higher than those in the world $(1.2 / 100,000$ and $0.7 / 100,000$, respectively), higher than those in East Asia (1.8/100,000 and $1.0 / 100,000$, respectively); the incidence higher but mortality lower than those in China in $2012(1.9 / 100,000$ and $1.2 / 100,000$, respectively). According to GLOBOCAN 2012, NPC incidence and mortality in China in 2013 ranked the 16th and 32th among 184 countries and regions in 2012, respectively; the incidence was similar to those in Thailand $(2.1 / 100,000)$ and Bhutan $(2.2 / 100,000)$, and the mortality was similar to those in Guam $(1.1 / 100,000)$ and Timor-Leste $(1.1 / 100,000)$. It indicated that NPC incidence and mortality in China in 2013 were still at high levels worldwide, although they both were lower than those in China in 2010 [1].

Compared with the study we carried out in 2014 [1], NPC incidence in China was lower in 2013 than in 2010, the crude incidence, ASIRC, and ASIRW decreased by $2.21,11.15$, and $11.07 \%$, respectively. The same results for the incidence were found in urban and rural areas. NPC crude mortality in China was slightly higher in 2013 than in 2010 (increased by $2.61 \%$ ), but the ASMRC and ASMRW were slightly lower in 2013 than in 2010 (decreased by $8.33 \%$ and $8.47 \%$, respectively). The same trends of mortality were found for rural areas. In urban areas, the rates were all lower in 2013 than in 2010. The incidences and mortalities were all not significantly different between the above 2 years. It revealed that comparing with the rates in 2010, NPC incidence and mortality in China in 2013 witnessed no obvious decreasing or increasing trend. This was not consistent with the results of the research by $\mathrm{Xu}$ et al. [13], which showed a decreasing mortality trend in China.

One of the epidemiological characteristics of NPC is that its incidence and mortality varied by geography, sex, and age. However, the present study revealed that there was no substantial difference in NPC incidence and mortality between Chinese urban and rural areas, which was basically consistent with the results in our previous study [1], suggesting that there were also no marked differences 
in factors linked to NPC incidence and mortality in urban and rural areas. Our study also showed that NPC incidence and mortality were the highest in the Western area, followed by the Middle and Eastern areas, but without significant differences, which was basically consistent with the results of previous results [1] and contrast to the results of the third national all-death-causes survey in China [5], and the reasons might be the same as those referred in our previous study [1], i.e., the distribution of sampling places may greatly influence the results. NPC geographic distribution features could be better described through stratification into seven administrative regions [1]. In the present study, NPC incidence and mortality were also significantly higher in South China than in other Chinese regions; the rates in South China were 3.4 and 3.8 times higher than those in the region with the second highest rates, respectively, and these findings were consistent with our previous reports too [1].

Our present study also showed that top 3 incidence provinces in 31 provinces, autonomous regions, and municipalities directly under the central government of China in 2013 were Guangxi, Guangdong, and Hunan, respectively, and top 3 mortality provinces were Guangxi, Guangdong, and Hainan, respectively-all locate in South China. It should be noticed that some provinces such as Tibet, Sichuan, Chongqing, Shanghai, and Hubei, which do not locate in South China, also ranked top 10 incidence and mortality provinces. Top 10 incidence and mortality areas among the 255 cancer registry areas in 2013 all locate in South China. Cangwu county of Guangxi autonomous region, Sihui city of Guangdong province, Longnan county of Jingxi province, and Mayang county of Hunan province were among the top 3 incidence or mortality areas. Zhongshan city of Guangdong province, which ranked the 1st in CIF 9 and 10 [23, 24 ], only ranked the 4th among the 255 cancer registry areas. However, Nanxiong city of Guangdong province and Longnan county of Jiangxi province, which are adjacent to each other geographically, also had high NPC incidence and mortality.

NPC incidence and mortality varied remarkably by sex and age. We determined that NPC incidence and mortality were significantly higher in males than in females, which was consistent with the results of previous reports [1-18]. Some differences existed between the age-specific incidences in the present study and our previous studies [1]. The female age-specific incidence peaked at age $80-84$ in the present study but at age $60-64$ in our previous study [1]. Difference in age-specific mortality between the present study and our previous study [1] was more obvious. The male and female age-specific mortalities were not close to each other at older age groups. The male age-specific mortalities increased quickly from age $30-34$, remained stable after age 60 , and peaked at age $80-84$ in our previous study [1], but increased quickly from $35-39$ and peaked at $80-84$ in the present study. The female age-specific mortalities increased more quickly after age 75-79 and peaked at age $85+$ in our previous study [1], but peaked at 75-79 and remained stable thereafter in the present study.

Although the data quality of NPC in this study were better than that in our previous study in 2014 [1], that in the 2012 Chinese Cancer Registry Annual Report [25, 26], and that of all cancers in 2013 in China [19, 20], the MV\% of NPC data in this study was still lower than that of NPC data in 2003-2007 Cancer Incidence and Mortality in China (79.14\%) [27], much lower than that of NPC data in CIF 9 (male, 96.28\%) [23] and CIF 10 (female, 83\%) [24], and also much lower than that of Zhongshan $[17,18]$. Moreover, NPC should have a high MV\%, as the nasopharynx is easy to be biopsied. All this indicated that NPC data quality in this study remained to be improved.

In conclusion, although NPC data quality in this study remained to be improved, the distribution or location of the selected population-based cancer registries could influence the results of the study, and the current results showed that NPC incidence and mortality in China in 2013 were still at high level worldwide, and these were consistent with the results of previous reports. NPC incidence and mortality in this study were close to the rates of our previous study in 2014, indicating that NPC incidence and mortality witnessed no obvious increasing or decreasing trend. Obvious differences of geography, sex, and age existed for the NPC incidence and mortality in this study, consistent with previous reports too. This study is very useful and helpful for the control and prevention of NPC in China, and enlightens the researches on NPC in the future.

\section{Authors' contributions}

KRW analyzed the data and wrote the paper. RSZ and SWZ prepared and calculated the data. ZHL and ZML data check and analysis, information retrieval. WQC conceived of the study and participated in its design and coordination. All authors read and approved the final manuscript.

\section{Author details}

${ }^{1}$ Cancer Institute of Zhongshan People's Hospital, Zhongshan, Guangdong 528403 , P. R. China. ${ }^{2}$ National Central Cancer Registry, National Cancer Center, Beijing 100021, P. R. China.

\section{Acknowledgements}

We acknowledge the cooperation of all the population-based cancer registries in providing cancer statistics, data collection, sorting, and verification, and database creation.

\section{Competing interests}

The authors declare that they have no competing interests.

Received: 8 May 2017 Accepted: 26 October 2017

Published online: 09 November 2017 


\section{References}

1. Wei KR, Zheng RS, Zhang SW, et al. Nasopharyngeal carcinoma incidence and mortality in China in 2010. Chin J Cancer. 2014;33(8):381-7.

2. Ferlay J, Soerjomataraml, Ervik M, et al. GLOBOCAN 2012 v1.0, Cancer Incidence and Mortality Worldwide: IARC CANCER BASE NO.11. Lyon: International Agency for Research on Cancer; 2013. http://globocan.iarc. fr. Accessed 18 Apr 2017.

3. Ferlay J, Bray F, Steliarova-Foucher E, et al. (2014) Cancer Incidence in Five Continents, Cl5plus. IARC Cancer Base No. 9. Lyon: International Agency for Research on Cancer, 2014. http://ci5.iarc.fr. Accessed 18 Apr 2017.

4. World Health Organization, Department of Information, Evidence and Research, mortality database. http://www-dep.iarc.fr/WHOdb/WHOdb. htm. Accessed 18 Apr 2017.

5. National Office for Cancer Control and Prevention, National Centre for Cancer Registration, Bureau for Disease Control and Prevention of Health Ministry. Chinese cancer mortality report-the third national death causes retrospective sampling survey. Beijing: People's Medical Publishing House; 2010. p. 171-87 (in Chinese).

6. Wei KR, Yu YL, Yang YY, et al. Epidemiological trends of nasopharyngeal carcinoma in China. Asian Pac J Cancer Prev. 2010:11(1):29-32.

7. Chua ML, Wee JT, Hui EP, et al. Nasopharyngeal carcinoma. Lancet. 2016:387(10022):1012-24

8. Wee JT, Soong YL, Chua ML. Nasopharyngeal carcinoma-past lessons and a glimpse into the future. Chin Clin Oncol. 2016;5(2):14

9. Carioli G, Negri E, Kawakita D, et al. Global trends in nasopharyngeal cancer mortality since 1970 and predictions for 2020: focus on low-risk areas. Int J Cancer. 2017;140(10):2256-64.

10. Tang $L L, C h e n W Q$, Xue WQ, et al. Global trends in incidence and mortality of nasopharyngeal carcinoma. Cancer Lett. 2016;374(1):22-30.

11. Safavi-Naini A, Raad N, Ghorbani J, et al. Incidence trends and geographical distribution of nasopharyngeal carcinoma in Iran. Iran J Cancer Prev. 2015;8(1):24-8

12. Arnold $M$, Wildeman MA, Visser $O$, et al. Lower mortality from nasopharyngeal cancer in The Netherlands since 1970 with differential incidence trends in histopathology. Oral Oncol. 2013;49(3):237-43.

13. $X u$ ZX, Lin ZX, Fang JY, et al. Mortality characteristic and prediction of nasopharyngeal carcinoma in China from 1991 to 2013. Asian Pac J Cancer Prev. 2015;16(15):6729-34.
14. Deng W, Lie JL, Yu JH, et al. Mortality trend of nasopharyngeal carcinoma in Guangxi Province. Yin Yong Yu Fang Yi Xue. 2011;17(2):73-6 (in Chinese).

15. Li K, Lin GZ, Shen JC, et al. Time trends of nasopharyngeal carcinoma in Urban Guangzhou over a 12-year period (2000-2011): declines in both incidence and mortality. Asian Pac J Cancer Prev. 2014;15(22):9899-903.

16. Zhang LF, LiYH, Xie SH, et al. Incidence trend of nasopharyngeal carcinoma from 1987 to 2011 in Sihui County, Guangdong Province, South China: an age-period-cohort analysis. Chin J Cancer. 2015;34:15.

17. Wei KR, XuY, Liu J, et al. No incidence trends and no change in pathological proportions of nasopharyngeal carcinoma in Zhongshanin1970-2007. Asian Pac J Cancer Prev. 2010;11(6):1595-9.

18. Wei KR, Liang ZH, Ou ZX. Nasopharyngeal carcinoma mortality in Zhongshan city of Canton in 1970-2010. Zhongguo Ai Zheng Za Zhi. 2014:24:241-5 (in Chinese)

19. Chen WQ, Zheng RS,Zhang SW, et al. Cancer incidence and mortality in China, 2013. Zhong Guo Zhong Liu, China Cancer. 2017;1-7 (in Chinese)

20. Chen WQ. ZhZysis based on urbanization level. Chin J Cancer Res. 2017;29(1):1-10.

21. World Health Organization. International Statistical Classification of Diseases and Related Health Problems 10th Revision (ICD-10)-2015-WHO Version for; 2015. http://apps.who.int/classifications/icd10/browse/2015/ en\#/C00-C14. Accessed 18 Apr 2017

22. National Cancer Center. Chinese Guideline for Cancer Registration. Beijing: Peoples Hygine Health Press; 2016. p.16-85 (in Chinese)

23. Curado MP, Edwards B, Shin HR, et al. Cancer incidence in five continents, vol. IX. Lyon: IARC Scientific Publications; 2007. p. 76.

24. Forman D, Bray F, Brewster DH, et al. Cancer incidence in five continents, vol. X. Lyon: IARC Scientific Publications; 2014. p. 105.

25. Hao J, Chen WQ. Chinese cancer registry annual report, vol. 17. Beijing: Military Medical Science Press; 2012. p. 44-7 (in Chinese).

26. Xu ZJ, Zheng RS, Zhang SW, et al. Nasopharyngeal carcinoma incidence and mortality in China in 2009. Chin J Cancer. 2013;32(8):453-60.

27. Zhao P, Chen WQ, Kong LZ. Chinese cancer incidence and mortality, 2003-2007, vol. 14. Beijing: Military Medical Science Press; 2012. p. 216-27 (in Chinese)

\section{Submit your next manuscript to BioMed Central and we will help you at every step:}

- We accept pre-submission inquiries

- Our selector tool helps you to find the most relevant journal

- We provide round the clock customer support

- Convenient online submission

- Thorough peer review

- Inclusion in PubMed and all major indexing services

- Maximum visibility for your research

Submit your manuscript at www.biomedcentral.com/submit
(OioMed Central 